\title{
E2F6/KDM5C promotes SF3A3 expression and bladder cancer progression through a specific hypomethylated DNA promoter
}

Kai-Long Liu®, Yue-Wei Yin, Bao-Sai Lu, Ya-Lin Niu, Dan-Dan Wang, Bei Shi, Hong Zhang, Ping-Ying Guo, Zhan Yang and Wei Li ${ }^{*}$ (1)

\begin{abstract}
Background: Abnormal expression of splicing factor 3 A subunit 3 (SF3A3), a component of the spliceosome, has been confirmed to be related to the occurrence and development of various cancers. However, the expression and function of SF3A3 in bladder cancer $(B C)$ remains unclear.

Methods: The SF3A3 mRNA and protein level were measured in clinical samples and cell lines by quantitative realtime PCR, Western blot and immunofluorescence staining. Evaluate the clinical correlation between SF3A3 expression and clinicopathological characteristics through statistical analysis in BC patients. The function of SF3A3 in BC cells was determined in vitro using MTT and colony analysis. Co-immunoprecipitation (CoIP) assay was used to detected E2F6 and $\mathrm{KDM} 5 \mathrm{C}$ interaction. Luciferase reporter and chromatin immunoprecipitation (ChIP) were used to examine the relationship between E2F6/KDM5C and SF3A3 expression.

Results: In the present study, we demonstrated that expression of SF3A3 was elevated in BC tissue compared to the normal bladder tissue. Importantly, the upregulation of SF3A3 in patients was correlated with poor prognosis. Additionally, overexpression of SF3A3 promoted while depletion of SF3A3 reduced the growth of BC cells in vivo and in vitro. Data from the TCGA database and clinical samples revealed that hypomethylation of the DNA promoter leads to high expression of SF3A3 in BC tissue. We found that upregulation of lysine-specific demethylase 5C (KDM5C) promotes SF3A3 expression via hypomethylation of the DNA promoter. The transcription factor E2F6 interacts with $\mathrm{KDM} 5 \mathrm{C}$, recruits $\mathrm{KDM} 5 \mathrm{C}$ to the SF3A3 promoter, and demethylates the $\mathrm{GpC}$ island of $\mathrm{H} 3 \mathrm{~K} 4 \mathrm{me} 2$, leading to high SF3A3 expression and $\mathrm{BC}$ progression.
\end{abstract}

Conclusions: The results demonstrated that depletion of the KDM5C/SF3A3 prevents the growth of BC in vivo and in vitro. The E2F6/KDM5C/SF3A3 pathway may be a potential therapeutic target for BC treatment.

Keywords: SF3A3, KDM5C, Hypomethylation, Proliferation, Bladder cancer

*Correspondence: Weili_hb2h@163.com

Department of Urology, The Second Hospital of Hebei Medical University,

215 Heping West Road, Shijiazhuang 050000, China

\section{Background}

Bladder cancer $(\mathrm{BC})$ is the most common malignant tumor among urinary system cancer. Statistically, there were 81,400 new cases of $\mathrm{BC}$ worldwide and more than 17,980 deaths in 2020 [1]. In China, the incidence and mortality of $\mathrm{BC}$ are at the forefront of genitourinary original author(s) and the source, provide a link to the Creative Commons licence, and indicate if changes were made. The images or other third party material in this article are included in the article's Creative Commons licence, unless indicated otherwise in a credit line to the material. If material is not included in the article's Creative Commons licence and your intended use is not permitted by statutory regulation or exceeds the permitted use, you will need to obtain permission directly from the copyright holder. To view a copy of this licence, visit http://creativecommons.org/licenses/by/4.0/. The Creative Commons Public Domain Dedication waiver (http://creativeco mmons.org/publicdomain/zero/1.0/) applies to the data made available in this article, unless otherwise stated in a credit line to the data. 
system tumors and are showing an upward trend [2]. More than $90 \%$ of $\mathrm{BC}$ is urothelial cell carcinoma, whereas squamous cell carcinoma accounts for about $5 \%$, and adenocarcinoma is rare [3]. Approximately $75 \%$ of the newly diagnosed cases are non-muscular invasive $\mathrm{BC}$ (NMIBC). Treatment methods generally include transurethral bladder tumor resection (TURBT), intravesical chemotherapy, and BCG immunotherapy [4, 5]. About $25 \%$ of cases belong to myometrial invasive BC (MIBC), which is highly malignant, prone to lymph node metastasis, and has a low 5-year survival rate [6]. Treatment methods generally include radical total cystectomy and urinary diversion [7]. Although there is some progress in the surgical treatment of $\mathrm{BC}$, immunotherapy, and combined radiotherapy and chemotherapy in recent years, the prognosis of invasive and metastatic $\mathrm{BC}$ has not been significantly improved [8]. These poor outcomes are due to insufficient understanding of the biological mechanisms of disease recurrence and progression [9]. Therefore, in-depth molecular mechanism research on genes closely related to its malignant progression can provide new ideas and directions for the diagnosis and treatment of BC.

The spliceosome is a multi-subunit complex composed of small non-coding RNAs (U1, U2, U4, U5, and U6) and a variety of related proteins [10]. Overwhelming evidence suggests that splicing is altered in many human tumors [11]. Previous studies have shown that transcriptional upregulation of several spliceosome components is an important means to ensure accurate pre-mRNA splicing and to protect the growth and survival of cancer cells [12]. Splicing factor 3A subunit 3 (SF3A3) is a component of the spliceosome, and it participates in the splicing of precursor mRNA as a component of the precatalytic spliceosome "B" complex [13, 14]). Studies have found that SF3A3 is involved in the occurrence and development of various tumors. The expression of $Y T H D F 2$ and $S F 3 A 3$ are positively correlated, and they play a synergistic role in the progression of hepatocellular carcinoma [15]. SF3A3 mediates cellular stress response to regulate tumor suppressor genes expression and cell death [16]. Recently, Maciej et al. showed that SF3A3 selectively regulates MYC-driven splicing and metabolic reprogramming, and the expression level of SF3A3 affects MYC-induced oncogenesis and breast cancer plasticity. However, the expression and role of SF3A3 in BC are still unclear.

Lysine-specific demethylase 5C (KDM5C), also known as JARID1C, is a member of the SMCY homolog family, which plays a key role in transcriptional regulation by H3K4me2/3 demethylation [17]. Studies have shown that abnormal expression of KDM5C is closely related to the occurrence and development of various cancers $[18,19]$.
Overexpression of KDM5c in human colon cancer cells leads to weakened $F B X W 7$ transcription and increases c-Jun protein, resulting in the proliferation of colon cancer cells [20]. In addition, KDM5C, which is highly expressed in $\mathrm{PCa}$ and CRPC, promotes the proliferation of castration-resistant prostate cancer (CRPC) cells through epigenetic inhibition of phosphatase and tensin homolog (PTEN) genes [21]. It is reported that KDM5C activates estrogen receptor alpha $(E R \alpha)$ target genes while inhibiting type I interferon (IFN) and interferon stimulating genes to promote the growth of ER $\alpha$-positive breast cancer cells and tumorigenesis [22]. Despite KDM5c is associated with a variety of cancer types, the function of KDM5c in the progression of $\mathrm{BC}$ is largely unknown.

In this study, we demonstrated that upregulation of SF3A3 in BC tissue was correlated with poor survival in $\mathrm{BC}$ patients. Moreover, overexpression of SF3A3 promoted while depletion of SF3A3 reduced the growth of $\mathrm{BC}$ cells. In addition, we found that hypomethylation of the DNA promoter leads to high expression of SF3A3 by upregulating $\mathrm{KDM} 5 \mathrm{C}$. Interestingly, we determined that E2F6 binds to KDM5C and recruits KDM5C to the GpC island of the SF3A3 promoter to induce histone demethylation. Depletion of the KDM5C/SF3A3 prevents BC progression. The E2F6/KDM5C/SF3A3 pathway may be a potential therapeutic target for $\mathrm{BC}$ treatment.

\section{Methods}

\section{Samples collection}

The human primary $\mathrm{BC}$ tissue and corresponding normal bladder tissue were collected from BC patients in the Second Hospital of Hebei Medical University from July 2017 to June 2021. All BC patients underwent curative radical cystectomy in urology department. The research protocol and sample collection were approved by the Ethics Committee of the Second Hospital of Hebei Medical University, and each patient signed a written informed consent [26].

\section{Cell culture and transfection}

UM-UC-3, 253 J, T24 and J82 cell lines were purchased from The Global Bioresource Center (ATCC, Maryland), and expanded and stored in our laboratory. The above cell were cultured with dulbecco's modified eagle medium (DMEM) (Gibco, USA) medium supplemented with $10 \%$ fetal bovine serum (Clark Bio, Claymont,DE, USA) and $1 \%$ penicillin/streptomycin (Gibco, USA). Cells were cultured under humidified conditions of $95 \%$ air and $5 \% \mathrm{CO}_{2}$. Cells were passaged at about $80 \%$ confluence. Vectors were transfected into the cells by using Lipofectamine 2000 (Invitrogen) according to the manufacturer's protocol [23]. The siRNAs such as shSF3A3, shKDM5C and shE2F6 and negative controls were 
synthesized by GenePharma Co., Ltd. SF3A3 (oeSF3A3), oeKDM5C, and oeE2F6 plasmids and lentiviral vectors were gained from GENEWIZ (Suzhou, China).

\section{RNA isolation and RT-qPCR}

The tissues or cells were lysed by using QIAzol Lysis Reagent (iagen), and then miRNeasy Mini Kit was used to isolation total RNA (217,004; Qiagen). NanoDrop 2000 (Thermo) was used for total RNA quality examination. First strand of cDNA was synthesized by using M-MLV First Strand Kit (Life Technologies) and random hexamer primers. Platinum SYBR Green qPCR Super Mix UDG kit (Invitrogen) were used to detect gene mRNA expression in the ABI 7500 FAST system (Life Technologies). The relative expression levels were normalized to GAPDH and results were analyzed by the $2^{-\Delta \Delta C t}$ formula [24]. The corresponding primers were listed in Additional file 1: Table S1.

\section{Western blot}

RIPA lysis buffer was used to extraction the proteins from cultured cells and frozen tissue clinical samples. Protein samples was separated in SDS-PAGE and then electrotransferred to PVDFmembrane (Millipore). The membrane was incubated with 5\% skimmed milk for $2 \mathrm{~h}$ and treatment with the primary antibody overnight at $4{ }^{\circ} \mathrm{C}$. The antibodies in this study as follows: anti- $\beta$ actin (1:1000, sc-47,778), anti-SF3A3 (1:1000, ab176581), anti-c-MYC (1:500, ab32072), anti-KDM5C (1:1000, ab259913), and anti-E2F6 (1:500, ab155978). Next day, HRP-conjugated secondary antibody (1:5000, Rockland) was used to reaction with the membrane. Western blots were processed by ECL (enhanced chemiluminescence, Millipore) and detected using Fuazon Fx (Vilber Lourmat). FusionCapt Advance Fx5 software (Vilber Lourmat) were used to capture and process images [25] (Additional file 2).

\section{Vector construction and luciferase reporter assay}

The 2-kb SF3A3 promoter sequence was inserted into the pGL3 basic vector after treatment with Mlu1 and Xho1. Sanger sequencing was used to confirm the sequence. BC cell lines were seeded into a 24-well plate, and SF3A3prom vector, oeKDM5C, or shE2F6 and the corresponding control vector were co-transfected to cells for $24 \mathrm{~h}$. Luciferase activity was measured by Dual-Glo Luciferase Assay System (Promega, Madison, WI, USA) with Flash and Glow (LB955, Berthold Technologies) [26].

\section{Xenograft animal model}

Approximately $18-22$ g male BALB/c nude mice (4-6 weeks old) were purchased from Vital River Laboratory Animal Technology Co., Ltd. (Beijing) and used for Xenograft animal mode. A total of $5 \times 10^{6} \mathrm{~T} 24$ cells stable shSF3A3/shKDM5C-infected were collected and resuspended in PBS and mixed with 50\% Matrigel (BD) to form a volume of $0.2 \mathrm{~mL}$ Inject the mixed suspension subcutaneously into the right dorsal side. One week later, the length and width of the mouse tumors were measured twice a week. Then, we used the following formula to calculate the tumor volume: tumor volume $=$ (length $\times$ width 2 )/2. After 28 days, the mice were euthanized by carbon dioxide asphyxiation $[23,25,27]$. The euthanasia protocol is as described previously [28]. The mice were placed in a transparent airtight box $(20 \mathrm{~cm} \times 12 \mathrm{~cm}$ $\times 12 \mathrm{~cm}$ ). A concentration of $99.9 \%$ carbon dioxide was injected into the closed box at a flow rate of $0.6 \mathrm{~L} / \mathrm{min}$ (approximately 20\% of the box volume). The mice were exposed to the carbon dioxide until a complete cessation of breathing, and observed for at least 2 min (usually about 5-10 min in total). Then, the mice were taken out of the box and treated with cervical dislocation to ensure death. Finally, xenograft tumor tissue were removed for later use.

\section{Morphometry and histology}

Morphometry and histology were detected by hematoxylin and eosin staining as described before [27, 29]. BC and corresponding normal tissues were fixed in formalin solution and then embedded in conventional paraffin. $5 \mu \mathrm{m}$ thick sections were used for hematoxylin and eosin staining or immunofluorescence staining. A Leica microscope (Leica DM6000B, Switzerland) was used to acquire sectional images, and LAS V.4.4 (Leica) was used for digitization.

\section{Immunofluorescence staining}

The above-mentioned embedded clinical sample tissue and xenograft tissue were sliced and routinely deparaffinized to water as described before [30]. The tissue sections were blocked with goat serum after antigen retrieval. These were incubated overnight with antiSF3A3 (ab176581) and anti-c-MYC (1:500, ab32072). The tissue was treated with fluorescein-labeled anti-rabbit IgG (KPL) and rhodamine-labeled anti-mouse IgG (KPL) [30]. DAPI (157,574; MB Biomedical) was used for nuclear staining. The images were captured using a confocal microscope (DM6000 CFS; Leica) and processed using LAS AF software $[27,29]$.

\section{MTS assay}

According to the product's instruction manual (ab197010), the cell viability was examined by the MTS. In brief, T24 and J82 cells were planted into 96-well plates and transfected with the corresponding vectors. Then, $20 \mu \mathrm{L}$ of MTS reagent were added to each well and incubated for $0.5-4 \mathrm{~h}$. 
Finally, the absorbance of the cells was detected by a microplate reader at $490 \mathrm{~nm}$ (Thermo Fisher, USA).

\section{Chromatin immunoprecipitation (ChIP) assay}

In order to explore the interaction of transcription factors with DNA, we performed chromatin immunoprecipitation (ChIP) as described above [31]. Cultured T24 cells were fixed with formaldehyde and neutralized with glycine to prepare cross-linked chromatin. After that DNA samples were sonicated to an average long of 400-600 bp. These samples were diluted and then incubated with protein A-Sepharose/Salmon sperm DNA for at $4{ }^{\circ} \mathrm{C}$. Then anti-KDM5C, anti-E2F6, or anti-IgG (as a negative control) antibody were used to immunoprecipitate DNA fragments. After the crosslinking was reversed, the binding rate of KDM5C and E2F6 on the SF3A3 promoter was checked. The result was confirmed by qRT-PCR.

\section{Colony formation assay}

In order to detected the cell proliferation the colony formation assay was performed [30]. 100 cells/well were seeded into a six-well plate and continuous cultured for 10-14 days. Then, these were fixed in methanol solution and colony was stained with $0.5 \%$ crystal violet. The number of colonies was counted under the microscope.

\section{Co-immunoprecipitation (CoIP) assay}

Cell lysates were immunoprecipitated by overnight incubation with the indicated antibody at $4{ }^{\circ} \mathrm{C}$, followed by another overnight incubation with protein $\mathrm{A}$-agarose for $1 \mathrm{~h}$. The protein A-agarose-antigen-antibody complexes were collected through centrifugation at 12,000 $\mathrm{g}$ for $2 \mathrm{~min}$ at $4{ }^{\circ} \mathrm{C}$, followed by washing five times with $1 \mathrm{~mL}$ of immunoprecipitation-HAT buffer for $20 \mathrm{~min}$ each time at $4{ }^{\circ} \mathrm{C}$ [24]. The bound proteins were resolved on an SDS-PAGE gel, followed by Western blotting using the corresponding antibody.

\section{Statistical analysis}

Statistical analysis as mentioned before [24]. Data were expressed as mean \pm SEM. The Student's t-test was used to analyze the difference between the two groups. Spearman correlation analysis was used to evaluate correlation analysis. A value of $\mathrm{P}<0.05$ was considered statistically significant. GraphPad Prism 7.0 software was used for statistical analysis (GraphPad Software) [30].

\section{Results}

The upregulation of SF3A3 contributes to poor prognosis of $B C$ patients

A previous study showed that SF3A3 facilitates breast cancer progression [32]. To explore the expression of SF3A3 in BC tissues, we first collected the clinical samples and confirmed tumor and normal tissues by using hematoxylin and eosin staining (Fig. 1A). Then, we examined the expression of SF3A3 in BC tissues by immunofluorescence staining. The results showed that the fluorescence intensity of SF3A3 significantly increase in $\mathrm{BC}$ tissue (Fig. 1B). The Western blot results also revealed that the SF3A3 protein level was significantly increased in BC tissues (Fig. 1C, D). By analyzing the mRNA expression in the clinical sample or TCGA data set, we demonstrated that expression of SF3A3 was markedly upregulated in $\mathrm{BC}$ tissue than in normal bladder tissue (Fig. 1E, F). In addition, the Kaplan-Meier correlation analysis in TCGA database also showed that patients with high SF3A3 expression in BC were predicted to have poorer overall survival (Fig. 1G). Furthermore, the clinicopathological factors of SF3A3 expression level were positively correlated with tumor size but did not correlate with other such as age, gender, and tumor grade (Table 1). Next, we then measured the expression of SF3A3 in BC cell lines and found that SF3A3 had a higher expression in T24 cells and lower expression in J82 cells (Fig. $1 \mathrm{H}-\mathrm{J}$ ). These results confirmed that SF3A3 is elevated in $\mathrm{BC}$ tissue and may be associated with $\mathrm{BC}$ progression.

\section{SF3A3 facilitates the growth of $B C$ cells in vitro}

To explore the function of SF3A3 in BC, we first constructed two short hairpin RNA (shRNA) targeting SF3A3 and knocked it out in T24 cells while overexpressing SF3A3 in J82 cells by transfecting with the pWPI-SF3A3 vector. The results showed that shSF3A3 RNAs transfection significantly downregulated while pWPI-SF3A3 vector transfection upregulated the SF3A3 and MYC protein expression but did not affect MYC mRNA level (Fig. 2A-C). Next, we performed the MTS and colony formation assay to measure cell viability. The results showed that depletion of SF3A3 significantly reduced T24 cell proliferation while overexpression of SF3A3 promoted cell growth (Fig. 2D-F). Together, these data reveal a function of SF3A3 in the regulation of cell growth in $\mathrm{BC}$.

\section{Upregulated KDM5C promotes SF3A3 expression via hypomethylation of the DNA promoter}

Studies showed that methylation dysfunction of gene promoters induces tumor progression [33,34]. To investigate whether abnormal expression of SF3A3 is regulated by DNA methylation, we first analyzed methylation modification of SF3A3 promoter from TCGA database and found that there is hypomethylation in SF3A3 promoter compared with normal bladder tissue (Fig. 3A, Additional file 2). Then, we analyzed the $\mathrm{GpC}$ island of SF3A3 promoter (Fig. 3B) and examined the methylation level 


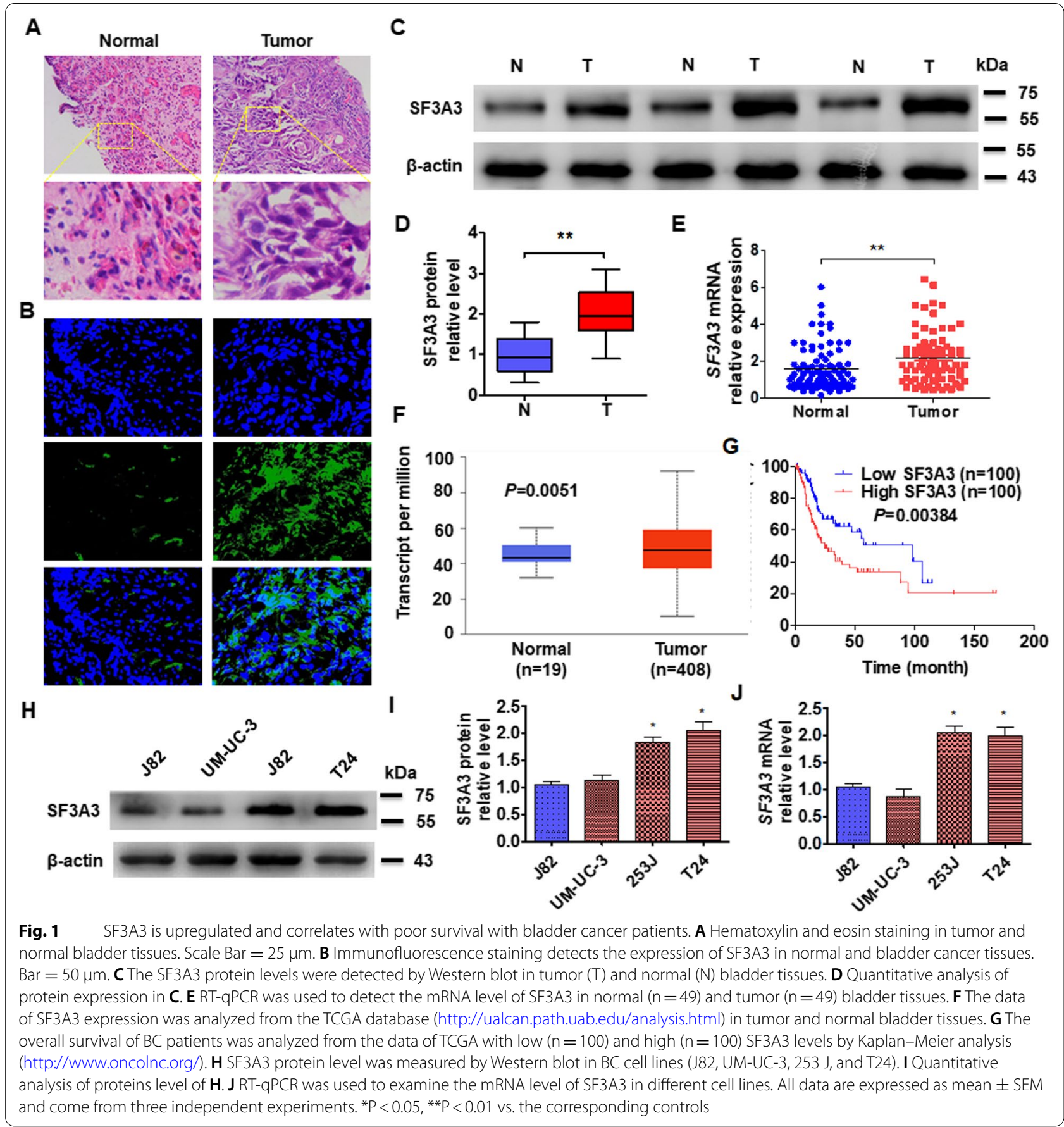

in GpC island using MSP and bisulfite sequencing PCR (BSP) analyses. The results revealed that the methylation level of SF3A3 CpG was lower in BC than that in normal bladder tissue (Fig. 3C). To investigate which demethylases may involve in SF3A3 promoter hypomethylation, we explored candidate genes in the clinical samples. As shown in Fig. 3D, KDM5C, KDM2B, and PCGF6 were upregulated and JMJD6 was downregulated in $\mathrm{BC}$ tissue. Next, siRNAs of these genes were synthesized and transfected into T24 cells, and then the expression of SF3A3 was examined by RT-qPCR. As showed in Fig. 3F, only knocking out KDM5C reduced SF3A3 expression in T24 cells. In addition, we detected the expression of SF3A3 in clinical samples and found that the expression of SF3A3 


\begin{tabular}{|c|c|c|c|c|}
\hline \multirow[t]{2}{*}{ Characteristic } & \multirow{2}{*}{$\begin{array}{l}\text { Number of } \\
\text { patients (\%) }\end{array}$} & \multicolumn{2}{|c|}{ SF3A3 expression } & \multirow[t]{2}{*}{ P value $^{a}$} \\
\hline & & Low (\%) & High (\%) & \\
\hline Patients & 82 & 42 & 40 & \\
\hline \multicolumn{5}{|l|}{ Age (years) } \\
\hline$\leq 60^{\mathrm{b}}$ & 34 & $18(52.86)$ & $16(47.14)$ & \multirow[t]{2}{*}{0.826} \\
\hline$>60$ & 48 & $24(50.55)$ & $24(49.45)$ & \\
\hline \multicolumn{5}{|l|}{ Gender } \\
\hline Male & 62 & $30(54.90)$ & $32(45.10)$ & \multirow[t]{2}{*}{0.445} \\
\hline Female & 20 & $12(45.76)$ & $8(54.24)$ & \\
\hline \multicolumn{5}{|l|}{ Tumor size (cm) } \\
\hline$\leq 3.0^{c}$ & 54 & $31(54.72)$ & $13(45.28)$ & \multirow[t]{2}{*}{0.014} \\
\hline$>3.0$ & 28 & $11(27.27)$ & $17(72.73)$ & \\
\hline \multicolumn{5}{|l|}{ Tumor grade } \\
\hline Low & 49 & $23(46.39)$ & $26(53.61)$ & \multirow[t]{2}{*}{0.376} \\
\hline High & 33 & $19(56.25)$ & $14(43.75)$ & \\
\hline \multicolumn{5}{|l|}{ T classification } \\
\hline $\mathrm{Ta}, \mathrm{T} 1$ & 53 & $31(43.81)$ & $22(56.19)$ & \multirow[t]{2}{*}{0.106} \\
\hline $\mathrm{T} 2-\mathrm{T} 4$ & 29 & $11(60.71)$ & $18(39.29)$ & \\
\hline \multicolumn{5}{|l|}{ pN status } \\
\hline $\mathrm{pN}-$ & 58 & $32(48.25)$ & $26(51.75)$ & \multirow[t]{2}{*}{0.334} \\
\hline $\mathrm{pN}+$ & 24 & $10(61.70)$ & $14(38.30)$ & \\
\hline \multicolumn{5}{|c|}{ Tumor multiplicity } \\
\hline Unifocal & 35 & $14(40.58)$ & $21(59.42)$ & \multirow[t]{2}{*}{0.118} \\
\hline Multifocal & 47 & $28(42.39)$ & $19(57.61)$ & \\
\hline
\end{tabular}

Significant associations are shown in bold in the $p$ value column ( $p$ value $<0.05$ )

${ }^{\text {a }}$ Chi-square test

${ }^{\mathrm{b}}$ Median age

${ }^{\text {cMedian size }}$

was increased in tumor tissue (Fig. 3F). Similar results were found in immunofluorescence staining (Fig. 3G, H). Otherwise, SF3A3 is also highly expressed in cells with high KDM5C expression (Fig. 3G). Furthermore, correlation analysis revealed that SF3A3 mRNA level was positively correlated with $K D M 5 C$ expression in $\mathrm{BC}$ tissue (Fig. 3I). Besides, the high expression of KDM5C in $\mathrm{BC}$ with patients was associated with a poor prognosis (Fig. 3J). These findings suggest that SF3A3 is a regulator modulated by KDM5C to drive tissue expansion.

\section{E2F6 interaction with KDM5C and boosts histone demethylation}

$\mathrm{T}$ figure out whether $K D M 5 C$ regulates SF3A3 expression by demethylating, we first overexpressed or depleted $K D M 5 C$ in cells and examined this gene expression. As shown in Fig. 4A, B, depletion of KDMSC reduced the expression of KDM5C and SF3A3 in T24 cells while overexpression of $K D M 5 C$ upregulates both proteins level. Similar results were found in RT-qPCR (Fig. 4C). To investigate whether $\mathrm{KDM} 5 \mathrm{C}$ regulates the histone methylation level, we transfected with oeKDM5C and detected the H3K4me2 methylation in the GpC island of SF3A3 promoter by ChIP. As expected, depletion of KDM5C significantly elevated the H3K4me2 methylation level in T24 cells (Fig. 4D). Conversely, overexpression of KDM5C markedly depressed the methylation level in the $\mathrm{GpC}$ island of the SF3A3 promoter (Fig. 4E). To identify how KDM5C regulated the methylation of SF3A3 specifically, we performed a CoIP -mass spectrometry (CoIP-MS). Seven proteins were found to enhance the interaction with KDM5C in KDM5C-overexpressing BC cells (Fig. 4F). CoIP coupled with Western blot confirmed that E2F6 interacted with KDM5C (Fig. 4G). Additionally, knocking out E2F6 would disrupt the methylation of the SF3A3 promoter, which was induced by overexpressing KDM5C in BC cells (Fig. 4H). Collectively, these data revealed that a function of $\mathrm{KDM} 5 \mathrm{C}$ is interaction with E2F6 and promotion of SF3A3 expression via histone demethylation.

\section{E2F6/KDM5C binds to the GpC island of the SF3A3 promoter and facilitates the growth of $\mathrm{BC}$ cells in vitro}

To investigate whether transcription factor E2F6 recruits $\mathrm{KDM} 5 \mathrm{C}$ to $\mathrm{GpC}$ island of the SF3A3 and regulates SF3A3 expression, we first analyzed the potential E2F6 binding site within the $\mathrm{GpC}$ island of SF3A3 promoter. As shown in Fig. 5A, there are two potential E2F6 binding elements. Next, ChIP analysis was performed and demonstrated that $\mathrm{E} 2 \mathrm{~F} 6 / \mathrm{KDM} 5 \mathrm{C}$ bound to the $\mathrm{GpC}$ island located - 744 to -665 bp within the SF3A3 promoter (Fig. 5B). To further confirm whether E2F6/KDM5C regulates the promoter activity of SF3A3, we performed the luciferase assay in $\mathrm{J} 82$ cells. The results revealed that KDM5C overexpression enhanced the luciferase activity of the SF3A3 promoter and this would be reversed by depleting GF1B simultaneously (Fig. 5C). These results revealed that E2F6/KDM5C binds to the GpC island of the SF3A3 promoter and regulates SF3A3expression. To examine the function of E2F6/KDM5C in BC cells, we performed rescue experiments. Overexpression of SF3A3 promoted the J82 cell growth and this would be reversed with depletion of KDM5C (Fig. 5D). In addition, knocking out KDM5C or E2F6 alone could depress the proliferation of T24 cells and depletion of both will enhance this effect (Fig. 5E). In parallel, the colony formation assays provided similar results (Fig. 5F, G). Collectively, these data suggest that 


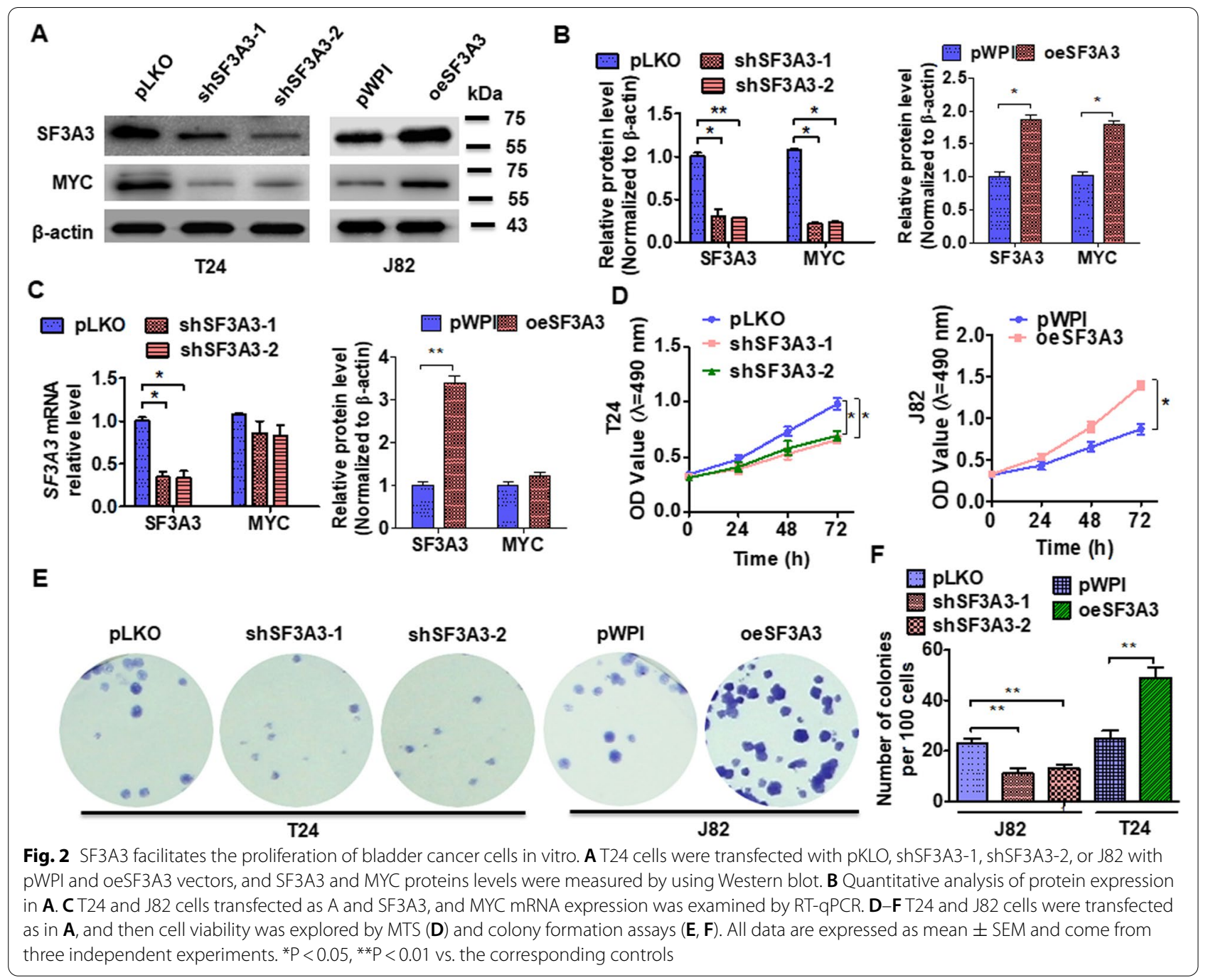

E2F6/KDM5C regulates cell growth by binding to the SF3A3 promoter.

\section{Interference with the E2F6/KDM5C/SF3A3 axis prevents the growth of $\mathrm{BC}$ xenografts in vivo}

To study whether disruption of the E2F6/KDM5C/ SF3A3 axis inhibits cell proliferation in vivo, a xenografts model was performed. As shown in Fig. 6A, knocking out SF3A3 or KDM5C alone results in smaller tumors compared to injection of control vector cells. In addition, the tumor volume and wet weights were much lower in mice implanted with SF3A3/KDM5C double depletion cells than that only single knocked-out cells (Fig. 6B, C). Double immunofluorescence staining from the tumor tissue demonstrated that depletion of either SF3A3 or KDM5C showed significantly reduced fluorescence intensity compared to control cells while this decrease was enhanced by deletion of both SF3A3 and KDM5C, simultaneously (Fig. 6D, E). Consistent with these, Western blot analysis of tumor tissue also yielded similar results (Fig. 6F, G). Taken together, our combined results indicated that blocking the E2F6/ KDM5C/SF3A3 axis inhibits the growth of BC xenografts in vivo.

\section{Discussion}

In the present study, we identified an E2F6/KDM5C/ SF3A3 axis that drives BC progression. Firstly, SF3A3 expression was significantly elevated in BC tissue compared to normal bladder tissue, and upregulation of 


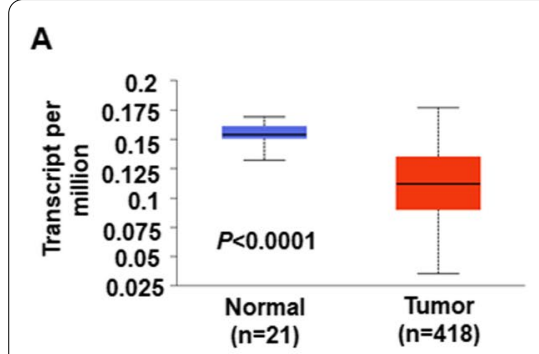

D

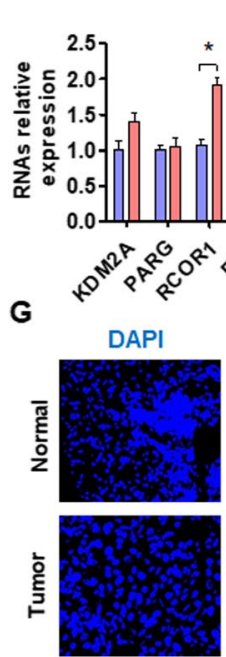

$\square$ Normal $\square$ Tumor
B

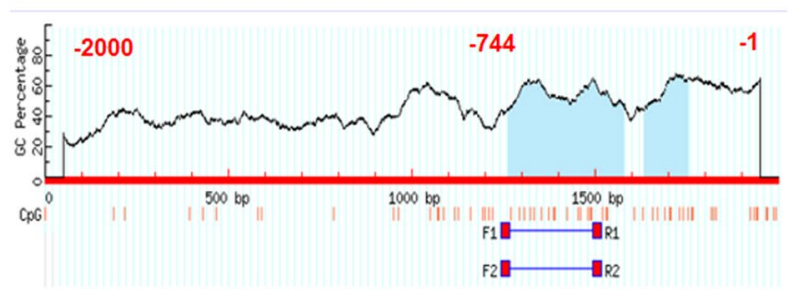

\section{C}

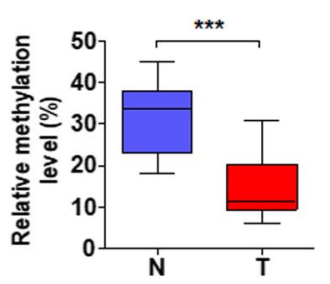

E

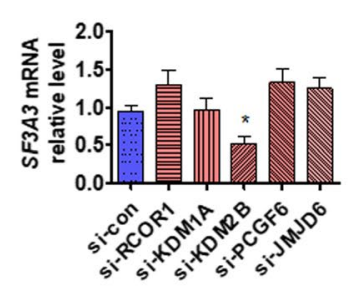

H

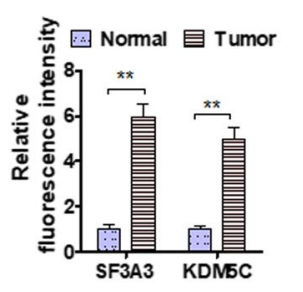

F

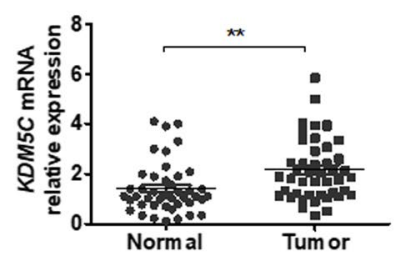

I

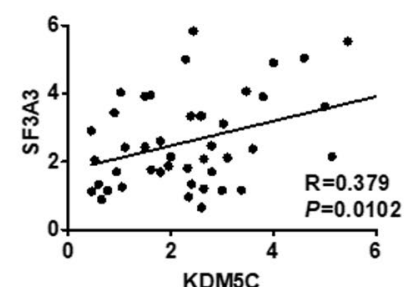

Fig. 3 Hypomethylation of promoter induces SF3A3 expression by upregulating KDM5C. A The DNA methylation level of SF3A3 promoter was explored from the TCGA database (http://ualcan.path.uab.edu/analysis.html) in tumor and normal bladder tissues. B The GpC island of SF3A3 promoter was analyzed by Methprimer (http://www.urogene.org/cgi-bin/methprimer/methprimer.cgi). C DNA methylation was measured using bisulfite sequencing PCR (BSP) in the GpC island of the SF3A3 promoter. D The expression of candidate DNA demethylases was measured by RT-qPCR in tumor and normal bladder tissues. ET24 cells were transfected with indicated siRNA, and then SF3A3 mRNA was detected by RT-qPCR. F RT-qPCR was used to explore the KDM5C expression in tumor and normal bladder tissues. G Double immunofluorescence staining was used to examine the expression of SF3A3 and KDM5C in tumor and normal bladder tissues. $\mathbf{H}$ Quantitative analysis of the fluorescence intensity SF3A3 and KDM5C. I Analysis of the correlation between SF3A3 and KDM5C expression in BC. J The overall survival of BC patients was analyzed with KDM5C levels by Kaplan-Meier analysis from the data of TCGA (http://www.oncolnc.org/). All data are expressed as mean \pm SEM and come from three independent experiments. ${ }^{*} P<0.05,{ }^{* *} P<0.01$ vs. the corresponding controls

SF3A3 in patients was correlated with poor prognosis. Secondly, overexpression of SF3A3 promoted and depletion of it inhibited BC cell proliferation in vivo and in vitro. Thirdly, the high expression of SF3A3 is caused by the hypomethylation of the DNA promoter driven by the upregulation of $\mathrm{KDM} 5 \mathrm{C}$ in $\mathrm{BC}$ tissue. In addition, transcription factor E2F5 bound and recruited KDM5C to the $\mathrm{GpC}$ island of the SF3A3 promoter and then promoted demethylation of H3K4me2. These findings suggest that the RE2F6/KDM5C/SF3A3 axis is a critical promoter of $\mathrm{BC}$ initiation and progression.

DNA methylation is one of the important chemical modifications of DNA involved in gene expression programming [35]. A large number of studies have shown that one of the hallmarks of cancer is abnormal DNA methylation [36]. In the past 20 years, the main research focus in this field has been the hypermethylation of tumor suppressor genes, but there is a relative lack of screening and research on hypomethylation genes for different cancers [37]. KDM5 family proteins include 4 subtypes of $\mathrm{KDM} 5 \mathrm{~A} / \mathrm{B} / \mathrm{C} / \mathrm{D}$, which catalyze the detrimethylation and dimethyl labeling of lysine 4 on histone H3 (H3K4), thereby regulating genes expression [38]. Depending on the methylation site, KDM5 can either activate or inhibit transcription [39]. New evidence suggests that the dysregulation of KDM5 is related to important phenotypic consequences of various types of cancer [40]. For example, KDM5C promotes the proliferation of 


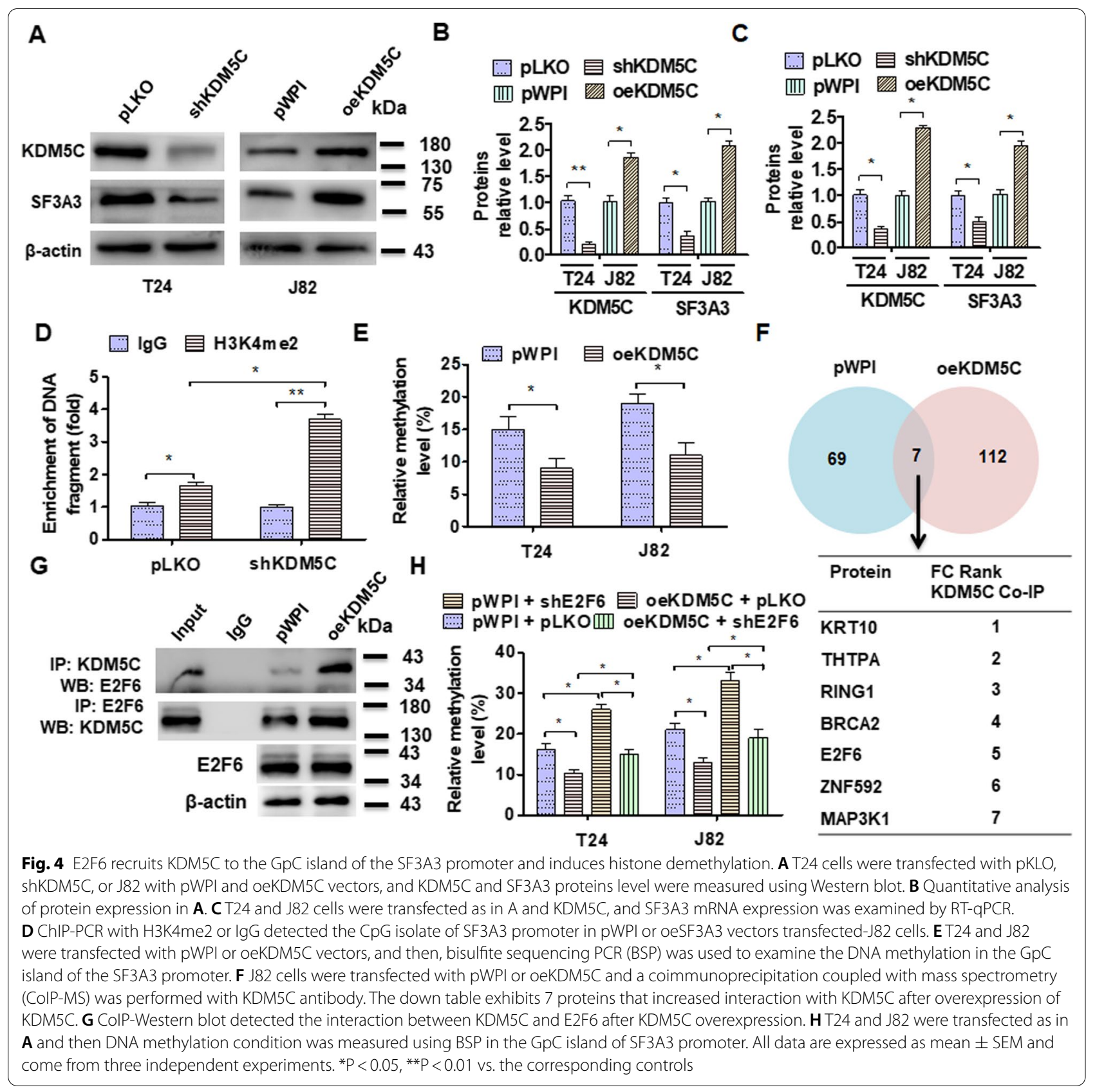

colon cancer cells through the FBXW7-c-Jun regulatory axis [20]. KDM5c inhibits the multidrug resistance of colon cancer cell lines by down-regulating $A B C C 1$ [41]. $K D M 5 C$ is transcriptionally regulated by $\mathrm{BRD} 4$ and promotes the proliferation of CRPC cells by inhibiting PTEN [21]. However, the expression and function of KDM5C in $\mathrm{BC}$ are still unclear. In this study, we found that KDM5C was highly expressed in $\mathrm{BC}$ tissue compared to normal bladder tissue, and upregulation of KDM5C promoted the hypomethylation of the DNA promoter in the $\mathrm{GpC}$ island of SF3A3. The hypomethylation of this promoter induced expression of SF3A3 and BC progression. Blocking of $\mathrm{KDM} 5 \mathrm{C}$ can reduce the cell proliferation of $\mathrm{BC}$ in vivo or in vitro.

Human splicing factor SF3A is an important part of $17 \mathrm{~S}$ U2 snRNP (small nuclear ribonucleoprotein 




particles). SF3A interacts with pre-mRNA branch sites to form an early spliceosome [42]. Using RNA to treat HeLa cells, the researchers found that removing individual subunits resulted in overall suppression of splicing, indicating that SF3A is a constitutive splicing factor [43]. SF3A includes three subtypes: SF3A1, SF3A2, and SF3A3. The structure and function analysis has clarified the relationship between the interaction of SF3A heterotrimer and the assembly of U2 snRNP and spliceosome [44]. Previous studies have shown that the transcriptional upregulation of several spliceosome components is an important means to ensure accurate pre-mRNA splicing and protect the growth and survival of cancer cells [12]. Recent studies have shown that the abnormal expression of SF3A3 is closely related to tumor progression [15]. SF3A3 selectively regulates MYC-driven splicing and metabolic reprogramming, which, in turn, induce tumorigenesis and breast cancer plasticity [32]. However, the expression of SF3A3 in BC and its function is largely unknown. In this study, we found that compared with normal bladder tissue, the expression of SF3A3 in BC tissue was significantly increased, and the upregulation of SF3A3 in patients was associated with a poor prognosis. In addition, the overexpression of SF3A3 promotes and deletion of it inhibits $\mathrm{BC}$ cell proliferation in vivo and 


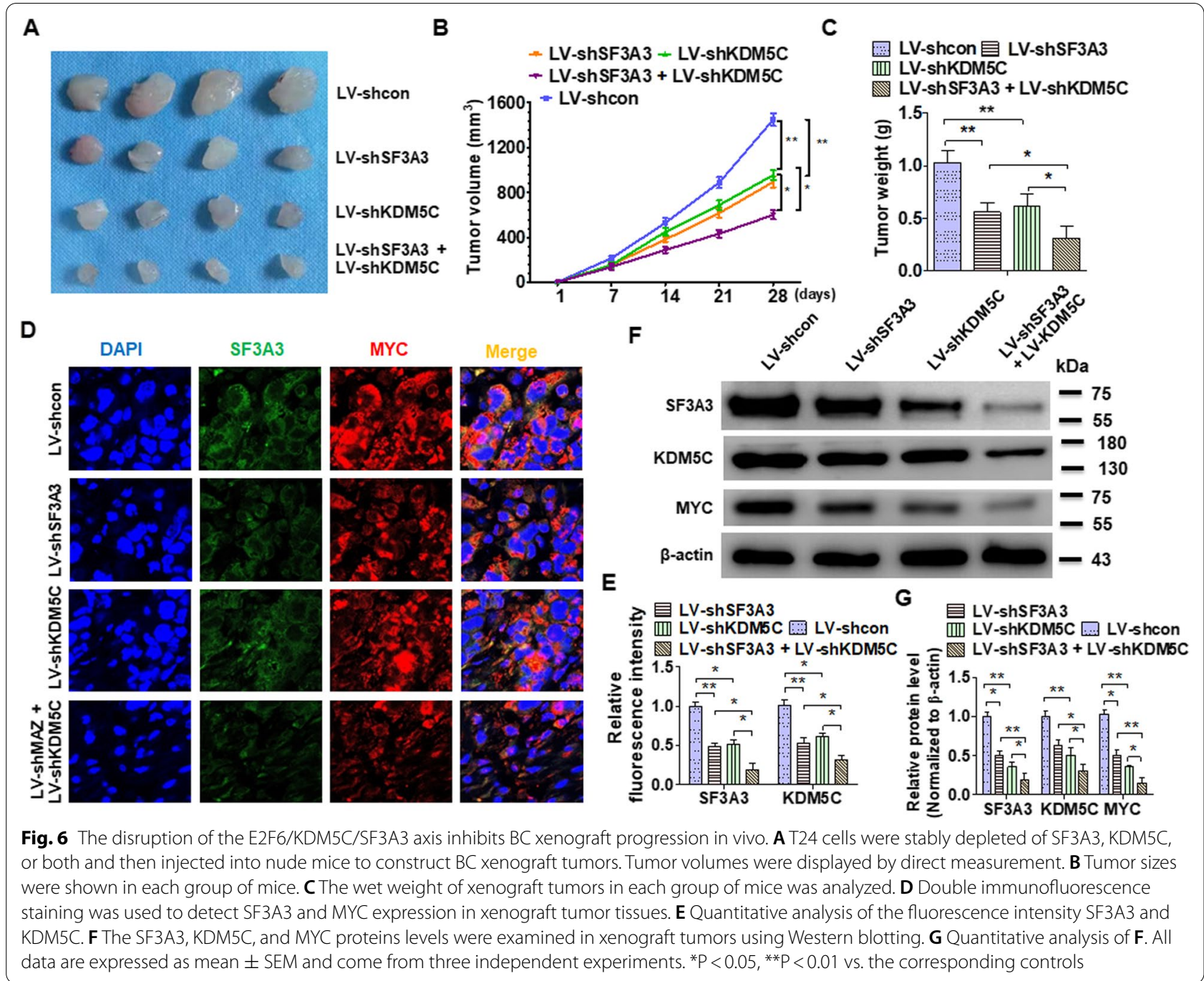

in vitro. Furthermore, we found that the high expression of SF3A3 is caused by the hypomethylation of the DNA promoter driven by the upregulation of KDM5C in $\mathrm{BC}$ tissue. Inhibition of either SF3A3 or KDM5C reduced the progression of $\mathrm{BC}$.

In conclusion, the present study reveals that elevation of SF3A3 promotes BC cell growth and is associated with poor $\mathrm{BC}$ prognosis. The upregulation of KDM5C was recruited by E2F6 to GpC island of SF3A3 promoter and demethylated $\mathrm{H} 3 \mathrm{~K} 4 \mathrm{me} 2$, then driving expression of SF3A3 and BC progression. These findings highlight that the $\mathrm{E} 2 \mathrm{~F} 6 / \mathrm{KDM} 5 \mathrm{C} / \mathrm{SF} 3 \mathrm{~A} 3$ axis is a potential therapeutic target for $\mathrm{BC}$ treatment.

\section{Supplementary Information}

The online version contains supplementary material available at https://doi. org/10.1186/s12935-022-02475-4.

\section{Additional file 1: Table S1. Oligos used in the study.}

Additional file 2: Fig. S1. Analyzed some genes positively related to SF3A3 expression in BC tissues from the TCGA database.

\section{Acknowledgements}

Not applicable.

\section{Authors' contributions}

Study conception and design: K-LL and WL. Acquisition of data: K-LL, BS, HZ and D-DW. Analysis and interpretation of data: K-LL, Y-WY and B-SL. Drafting of manuscript: K-LL and P-YG. Final approval of the submitted manuscript: all authors. All authors read and approved the final manuscript.

\section{Funding}

This study was partially supported by The National Natural Science Foundation of China (No. 81972410). The Natural Science Foundation of Hebei Province (No. H2019206257 and H2021206155).

Availability of data and materials Not applicable. 


\section{Declarations}

Ethics approval and consent to participate

The study protocol was approved by the Ethics Committee of the Second Hospital of Hebei Medical University, and written consent was obtained from each patient.

\section{Consent for publication}

Not applicable.

\section{Competing interests}

All authors declare no competing interests.

Received: 15 November 2021 Accepted: 18 January 2022

Published online: 05 March 2022

\section{References}

1. Siegel RL, Miller KD, Jemal A. Cancer statistics, 2020. CA Cancer J Clin. 2020;70:7-30

2. Wu P, Zhang G, Zhao J, Chen J, Chen Y, Huang W, et al. Profiling the urinary microbiota in male patients with bladder cancer in China. Front Cell Infect Microbiol. 2018;8:167.

3. Jordan B, Meeks JJ. T1 bladder cancer: current considerations for diagnosis and management. Nat Rev Urol. 2019;16:23-34.

4. Lenis AT, Lec PM, Chamie K, Mshs MD. Bladder Cancer: A Review. JAMA 2020;324:1980-1991.

5. Han J, Gu X, LiY, Wu Q. Mechanisms of BCG in the treatment of bladder cancer-current understanding and the prospect. Biomed Pharmacother. 2020;129:110393.

6. Chou R, Selph SS, Buckley DI, Gustafson KS, Griffin JC, Grusing SE, et al. Treatment of muscle-invasive bladder cancer: a systematic review. Cancer. 2016;122:842-51.

7. Patel VG, Oh WK, Galsky MD. Treatment of muscle-invasive and advanced bladder cancer in 2020. CA Cancer J Clin. 2020;70:404-23.

8. Martinez Rodriguez RH, Buisan Rueda O, Ibarz L. Bladder cancer: present and future. Med Clin (Barc). 2017;149:449-55.

9. Lu BS, Yin YW, Zhang YP, Guo PY, Li W, Liu KL. Upregulation of NPL4 promotes bladder cancer cell proliferation by inhibiting DXO destabilization of cyclin D1 mRNA. Cancer Cell Int. 2019;19:149.

10. Love MI, Huber W, Anders S. Moderated estimation of fold change and dispersion for RNA-seq data with DESeq2. Genome Biol. 2014;15:550.

11. Crews LA, Balaian L, Delos Santos NP, Leu HS, Court AC, Lazzari E, et al. RNA splicing modulation selectively impairs leukemia stem cell maintenance in secondary human AML. Cell Stem Cell. 2016;19:599-612.

12. Koh CM, Bezzi M, Low DH, Ang WX, Teo SX, Gay FP, et al. MYC regulates the core pre-mRNA splicing machinery as an essential step in lymphomagenesis. Nature. 2015;523:96-100.

13. Zhan $X$, Yan C, Zhang $X$, Lei J, Shi Y. Structures of the human pre-catalytic spliceosome and its precursor spliceosome. Cell Res. 2018;28:1129-40.

14. Zhang X, Yan C, Zhan X, Li L, Lei J, Shi Y. Structure of the human activated spliceosome in three conformational states. Cell Res. 2018;28:307-22.

15. Shao XY, Dong J, Zhang H, Wu YS, Zheng L. Systematic analyses of the role of the reader protein of $\mathrm{N}(6)$-methyladenosine RNA methylation, YTH domain family 2, in liver hepatocellular carcinoma. Front Mol Biosci. 2020;7:577460.

16. Zuo ZH, Yu YP, Martin A, Luo JH. Cellular stress response 1 down-regulates the expression of epidermal growth factor receptor and platelet-derived growth factor receptor through inactivation of splicing factor 3A3. Mol Carcinog. 2017;56:315-24.

17. Iwase S, Lan F, Bayliss P, de la Torre-Ubieta L, Huarte M, Qi HH, et al. The X-linked mental retardation gene SMCX/JARID1C defines a family of histone H3 lysine 4 demethylases. Cell. 2007;128:1077-88.

18. Carmignac V, Nambot S, Lehalle D, Callier P, Moortgat S, Benoit V, et al. Further delineation of the female phenotype with KDM5C disease causing variants: 19 new individuals and review of the literature. Clin Genet. 2020:98:43-55.
19. Chang S, Yim S, Park H. The cancer driver genes IDH1/2, JARID1C/ KDM5C, and UTX/ KDM6A: crosstalk between histone demethylation and hypoxic reprogramming in cancer metabolism. Exp Mol Med. 2019;51:1-17.

20. Lin H, Ma N, Zhao L, Yang G, Cao B. KDM5C promotes colon cancer cell proliferation through the FBXW7-c-Jun regulatory axis. Front Oncol. 2020;10:535449.

21. Hong Z, Wu G, Xiang ZD, Xu CD, Huang SS, Li C, et al. KDM5C is transcriptionally regulated by BRD4 and promotes castration-resistance prostate cancer cell proliferation by repressing PTEN. Biomed Pharmacother. 2019;114:108793

22. Shen HF, Zhang WJ, Huang Y, He YH, Hu GS, Wang L, et al. The dual function of $\mathrm{KDM} 5 \mathrm{C}$ in both gene transcriptional activation and repression promotes breast cancer cell growth and tumorigenesis. Adv Sci (Weinh). 2021:8:2004635.

23. Yang Z, Qu CB, Zhang Y, Zhang WF, Wang DD, Gao CC, et al. Dysregulation of p53-RBM25-mediated circAMOTL1L biogenesis contributes to prostate cancer progression through the circAMOTL1L-miR-193a-5p-Pcdha pathway. Oncogene. 2019:38:2516-32.

24. Ma Y, Zheng B, Zhang XH, Nie ZY, Yu J, Zhang H, et al. circACTA2 mediates Ang Il-induced VSMC senescence by modulation of the interaction of ILF3 with CDK4 mRNA. Aging. 2021;13:11610-28.

25. Yang Z, Chen JS, Wen JK, Gao HT, Zheng B, Qu CB, et al. Silencing of miR-193a-5p increases the chemosensitivity of prostate cancer cells to docetaxel. J Exp Clin Cancer Res. 2017;36:178.

26. Ren LX, Qi JC, Zhao AN, Shi B, Zhang H, Wang DD, et al. Myc-associated zinc-finger protein promotes clear cell renal cell carcinoma progression through transcriptional activation of the MAP2K2-dependent ERK pathway. Cancer Cell Int. 2021;21:323.

27. Yin YW, Liu KL, Lu BS, Li W, Niu YL, Zhao CM, et al. RBM24 exacerbates bladder cancer progression by forming a Runx1t1/TCF4/miR-625-5p feedback loop. Exp Mol Med. 2021:53:933-46.

28. Steven Leary WU, Raymond A, Samuel C, et al. AVMA Guidelines for the Euthanasia of Animals: 2020 Edition. American Veterinary Medical Association 2020:121.

29. Yang Z, Zheng B, Zhang Y, He M, Zhang XH, Ma D, et al. miR-155-dependent regulation of mammalian sterile 20-like kinase 2 (MST2) coordinates inflammation, oxidative stress and proliferation in vascular smooth muscle cells. Biochim Biophys Acta. 2015;1852:1477-89.

30. Qi JC, Yang Z, Lin T, Ma L, Wang YX, Zhang Y, et al. CDK13 upregulationinduced formation of the positive feedback loop among circCDK13, miR-212-5p/miR-449a and E2F5 contributes to prostate carcinogenesis. J Exp Clin Cancer Res. 2021;40:2.

31. Sun $Y$, Yang Z, Zheng B, Zhang XH, Zhang ML, Zhao XS, et al. A novel regulatory mechanism of smooth muscle alpha-actin expression by NRG-1/circACTA2/miR-548f-5p axis. Circ Res. 2017;121:628-35.

32. Ciesla M, Ngoc PCT, Cordero E, Martinez AS, Morsing M, Muthukumar S, et al. Oncogenic translation directs spliceosome dynamics revealing an integral role for SF3A3 in breast cancer. Mol Cell. 2021;81:1453-68 e1412.

33. Hoffman AM, Cairns P. Epigenetics of kidney cancer and bladder cancer. Epigenomics. 2011;3:19-34

34. Enokida H, Nakagawa M. Epigenetics in bladder cancer. Int J Clin Oncol. 2008;13:298-307.

35. Seisenberger S, Peat JR, Hore TA, Santos F, Dean W, Reik W. Reprogramming DNA methylation in the mammalian life cycle: building and breaking epigenetic barriers. Philos Trans R Soc Lond B Biol Sci. 2013;368:20110330.

36. Klutstein M, Nejman D, Greenfield R, Cedar H. DNA methylation in cancer and aging. Cancer Res. 2016;76:3446-50.

37. Stefanska B, Huang J, Bhattacharyya B, Suderman M, Hallett M, Han ZG, et al. Definition of the landscape of promoter DNA hypomethylation in liver cancer. Cancer Res. 2011;71:5891-903.

38. Plch J, Hrabeta J, Eckschlager T. KDM5 demethylases and their role in cancer cell chemoresistance. Int J Cancer. 2019;144:221-31.

39. Vinogradova M, Gehling VS, Gustafson A, Arora S, Tindell CA, Wilson C, et al. An inhibitor of KDM5 demethylases reduces survival of drug-tolerant cancer cells. Nat Chem Biol. 2016;12:531-8.

40. Wang GG, Song J, Wang Z, Dormann HL, Casadio F, Li H, et al. Haematopoietic malignancies caused by dysregulation of a chromatin-binding PHD finger. Nature. 2009;459:847-51. 
41. Lin H, Yang G, Yu J, Wang J, Li Q, Guo S, et al. KDM5C inhibits multidrug resistance of colon cancer cell line by down-regulating ABCC1. Biomed Pharmacother. 2018;107:1205-9.

42. Tanackovic G, Kramer A. Human splicing factor SF3a, but not SF1, is essential for pre-mRNA splicing in vivo. Mol Biol Cell. 2005;16:1366-77.

43. Kramer A, Ferfoglia F, Huang CJ, Mulhaupt F, Nesic D, Tanackovic G. Structure-function analysis of the U2 snRNP-associated splicing factor SF3a. Biochem Soc Trans. 2005;33:439-42.

44. Lin PC, Xu RM. Structure and assembly of the SF3a splicing factor complex of U2 snRNP. EMBO J. 2012;31:1579-90.

\section{Publisher's Note}

Springer Nature remains neutral with regard to jurisdictional claims in published maps and institutional affiliations.

- fast, convenient online submission

- thorough peer review by experienced researchers in your field

- rapid publication on acceptance

- support for research data, including large and complex data types

- gold Open Access which fosters wider collaboration and increased citations

- maximum visibility for your research: over $100 \mathrm{M}$ website views per year

At BMC, research is always in progress.

Learn more biomedcentral.com/submissions 